\title{
Unquenched Studies Using the Truncated Determinant Algorithm
}

\author{
A. Duncan ${ }^{\mathrm{a}}$, E. Eichten ${ }^{* b}$ and H. Thacker ${ }^{\mathrm{c}}$

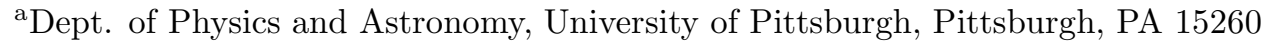 \\ ${ }^{\mathrm{b}}$ Fermilab, PO Box 500, Batavia, IL 60510 \\ ${ }^{c}$ Dept. of Physics, University of Virginia, Charlottesville, VA 22901
}

A truncated determinant algorithm is used to study the physical effects of the quark eigenmodes associated with eigenvalues below $420 \mathrm{MeV}$. This initial high statistics study focuses on coarse $\left(6^{4}\right)$ lattices (with $\mathrm{O}\left(a^{2}\right)$ improved gauge action), light internal quark masses and large physical volumes. Three features of full QCD are examined: topological charge distributions, string breaking as observed in the static energy and the eta prime mass.

\section{TDA Simulations on Coarse Lattices}

In the truncated determinant approach (TDA) to full QCD, the quark determinant, $\mathcal{D}(A)=$ $\operatorname{Det}(H)=\operatorname{Det}\left(\gamma_{5}(\not D(A)-m)\right)$ is split-up gauge invariantly into an infrared part and an ultravio-

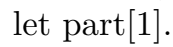

$\mathcal{D}(A)=\mathcal{D}_{I R}(A) \mathcal{D}_{U V}(A)$

The ultraviolet part, $\mathcal{D}_{U V}$, can be accurately fit by a linear combination of a small number of Wilson loops [2]. The infrared part $\mathcal{D}_{I R}(A)$ is defined as the product of the lowest $N_{\lambda}$ positive and negative eigenvalues of $H$, with $\left|\lambda_{i}\right| \leq \Lambda_{Q C D}$. The eigenvalues $\lambda_{a}$ of $H$ are gauge invariant and measure quark off-shellness. The cutoff (for the separation in Eq. 1) is tuned to include as much as possible of the important low-energy chiral physics of the unquenched theory while leaving the fluctuations of $\ln \mathcal{D}_{I R}$ of order unity after each sweep updating all links with the pure gauge action (assuring a tolerable acceptance rate for the accept/reject stage) [1,3]. This procedure works well even for kappa values arbitrarily close to kappa critical.

Initial studies using TDA focus on the qualitative physical effects of the inclusion of the infrared quark eigenmodes. For this purpose, coarse lattices with large physical volumes are appropriate. Here we report the study of $6^{4}$ lattices with an

*Presenter
$\mathrm{O}\left(a^{2}\right)$ improved gauge action

$$
\begin{aligned}
S_{G}= & \beta_{\text {plaq }} \sum_{\text {plaq }} \frac{1}{3} \operatorname{Re} \operatorname{Tr}\left(1-\mathrm{U}_{\text {plaq }}\right) \\
& +\beta_{\text {trt }} \sum_{\text {trt }} \frac{1}{3} \operatorname{Re} \operatorname{Tr}\left(1-\mathrm{U}_{\text {trt }}\right)
\end{aligned}
$$

with $\beta_{\text {plaq }}=3.7$ and $\beta_{\text {trt }} / \beta_{\text {plaq }}=1.04$. This $O\left(a^{2}\right)$ improved gauge action was adjusted (as in Ref. (4]) to have the lattice scale $a \approx 0.4 \mathrm{fm}$.

We used naive Wilson fermions with light quark masses ( $\kappa=0.2050,0.2044$ and 0.2038$)$; and keep the lowest 840 eigenvalues of $\mathcal{D}$, i.e. $\Lambda_{\text {cut }} \approx 420$ $\mathrm{MeV}(1 / a=.5 \mathrm{GeV})$. No terms modelling $\mathcal{D}_{U V}$ were included in the gauge action.

With $\kappa=.2050$, approximately 15,000 full steps were required for the lattice configurations to equilibrate (reflecting critical slowing down a few hundred suffice on small physical volumes). The equilibrated behaviour of $\mathcal{D}_{I R}$ is shown for 50,000 sweeps in Figure 1.

In the remaining sections we consider some physical quantities that differentiate between quenched and full QCD.

\section{Topolopy}

The topological charge, $Q$, can be expressed in terms of the eigenvalues of the Wilson-Dirac 


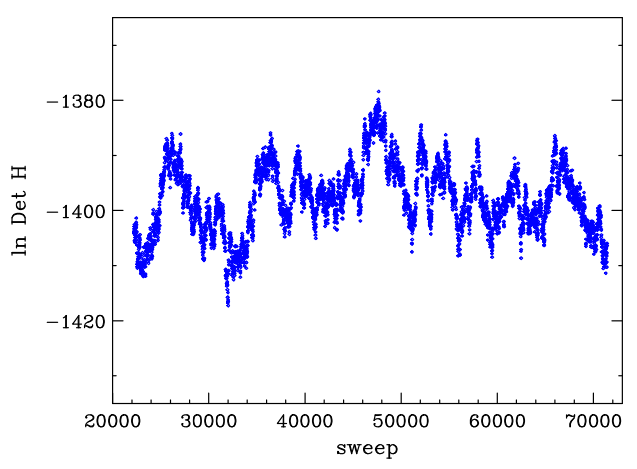

Figure 1. Equilibrated quark determinant in TDA simulations on a coarse $6^{4}$ lattice. $(\kappa=$ .2050) The lowest 840 eigenvalues included in ln Det $H$.

operator.

$Q=\frac{1}{2 \kappa}\left(1-\frac{\kappa}{\kappa_{c}}\right) \sum_{i=1}^{N} \frac{1}{\lambda_{i}}$

This sum is quickly saturated by the low eigenvalues.

In full QCD configurations very small eigenvalues of $H$ are suppressed by the quark determinant factor. In particular, non-zero topological charges must be suppressed in the chiral limit $\left(m_{q} \rightarrow 0\right)$. Furthermore, the functional dependence of the topological charge distribution, $P_{Q}$, on the light quark mass $m_{q}$ is predicted by the chiral analysis of Leutwyler and Smilga|[5].

$P_{Q}=I_{Q}(x)^{2}-I_{Q+1}(x) I_{Q-1}(x)$

where $x=1 / 2 V f_{\pi}^{2} m_{\pi}^{2}=V m_{q}<\bar{\psi} \psi>, I_{Q}$ are modified Bessel Functions of order $\mathrm{Q}$ and $\mathrm{V}$ is the total space-time volume.

The qualitative agreement with the expected behaviour of Eq. ¿4 has been reported previously[1]. Using the value of $x=3.8$ determined from the lattice calculations $\left(m_{\pi}=.39\right.$ and $f_{\pi}=.20$ for $\kappa=.2050$ ), excellent quantitative agreement between theory and lattice calculations is shown in Figure 2. The data from a single kappa value (e.g. $\kappa=.2050)$ determines

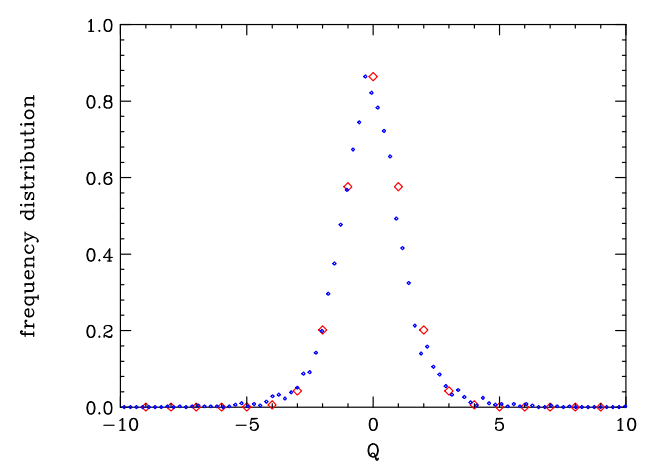

Figure 2. Topological charge distribution for $\kappa=.2050$ on a coarse $6^{4}$ lattice (dots). Expected chiral behaviour (diamonds) is also shown.

$\kappa_{c}$. Equating the calculated and predicted behaviour we find the factor $1-\kappa / \kappa_{c}=0.0082$, i.e. $\kappa_{c}=.2067$.

\section{Static Energy}

The static energy $V(\vec{R})$ is defined as:

$V(\vec{R}) \equiv \lim _{T \rightarrow \infty} \ln \frac{W(\vec{R}, T-1)}{W(\vec{R}, T)}$

In Coulomb gauge, $W(\vec{R}, T)$ is the Wilson line correlator and $V(R)$ is the energy of the lowest physical state coupling the static quark-antiquark state (with separation $R$ ) to the vacuum. As $T \rightarrow$ $\infty, \mathrm{V}(\mathrm{R})$ is approached from above.

Light quark masses implies long autocorrelations in physical quantities. However, with a bin size of 250 sweeps, the values of $W(\overrightarrow{(} R), T)$ are decorrelated for all relevant $R$ and $T[3]$.

The results for the static energy $(\kappa=0.2050)$ are shown in Figure 3. Clear evidence for string breaking is seen for $T \geq 3$. This strengthens previous indications for string breaking 3 [.

We can also extract the Sommer scale $\left(r_{0}^{2}[V(r)-V(r-d)] /|d|=1.65\right)$ from our determination of the static energy. Very roughly, $r_{0} \approx 1.25$ and $1 / a \approx 500 \mathrm{MeV}$. 


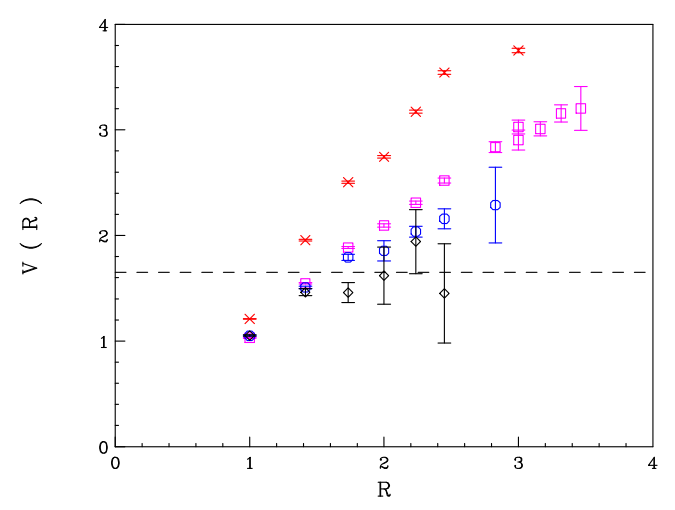

Figure 3. Static energy measured using time slices: [0:1] (x's), [1:2] (squares), [2:3] (circles) and [3:4] (diamonds). $(\kappa=0.2050)$ The heavylight pair threshold is denoted by the dashed line.

\section{Spectrum of Light Hadrons}

Masses for the light hadrons can be determined for the various kappa values. For $\kappa=.2050$ (highest statistics) we obtain: $m_{\pi}=0.391(6)$, $m_{\rho}=1.008(8)$ and $m_{P}=1.935(89)$. Significant scale violation is seen with naive Wilson fermions. For example, the scale $1 / a$ determined from the proton mass is $485 \mathrm{MeV}$ while the rho mass gives $764 \mathrm{MeV}$.

The relation between the axial $U(1)$ anomaly and the $\eta^{\prime}$ mass is well understood in full QCD. For two light quarks $\left(N_{f}=2\right), m_{\eta}^{2}=m_{\pi}^{2}+m_{0}^{2}$ where $m_{0}^{2}=2 N_{f} \chi / f_{\pi}^{2}$ and the topological susceptibility is $V \chi \equiv<Q^{2}>_{\text {quenched. }}$. The full $\eta$ propagator is the sum of a connected (valence quark) term and a disconnected (hairpin) term. Thus, in the continuum, the momentum space full propagator can be written:

$$
\begin{aligned}
\left(p^{2}+m_{\pi}^{2}+m_{0}^{2}\right)^{-1} & =\left(p^{2}+m_{\pi}^{2}\right)^{-1}- \\
& m_{0}^{2}\left(p^{2}+m_{\pi}^{2}\right)^{-1}\left(p^{2}+m_{\pi}^{2}+m_{0}^{2}\right)^{-1}
\end{aligned}
$$

These separate terms and their sum are shown in Figure 1 for $\kappa=0.2050$. The cancellation between the valence and hairpin terms in the full propagator is evident. We obtain $m_{\eta^{\prime}}=1.16(12)$. As expected the $\eta^{\prime}$ mass is much heavier than the pion mass.

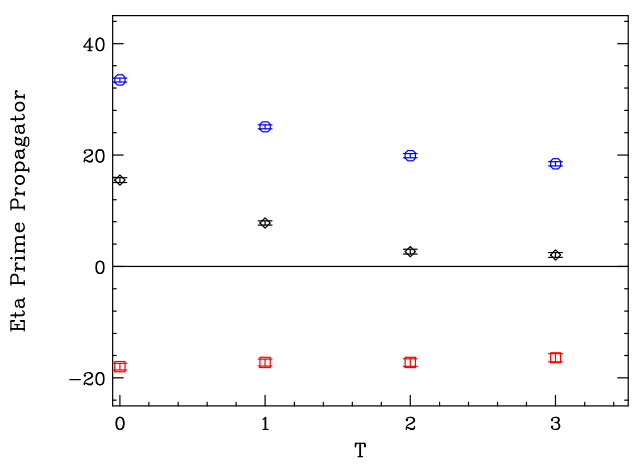

Figure 4. The total $\eta^{\prime}$ propagator for $\kappa=.2050$ on the $6^{4}$ lattices. The valence term (circles), hairpins term (squares) and total propagator (diamonds) are shown separately.

\section{Summary and Outlook}

Three results are clear from this study: (1) The topological charge distribution behaves as expected in full QCD. (2) Static energy shows clear evidence for string breaking for $\mathrm{T}>1 \mathrm{fm}$. (3) Light hadron spectrum results on a $6^{4}$ coarse lattice look reasonable. In particular, $m_{\eta^{\prime}} a=$ $1.16(12) \gg m_{\pi} a=.391(6)$.

The full parametric studies needed to optimize with TDA method will require many runs on small sets of CPU's. This might be done effectively by a seti@home approach [6].

\section{REFERENCES}

1. A. Duncan, E. Eichten and H. Thacker, Phys. Rev. D59, 014505 (1998).

2. A. Duncan, E. Eichten, R. Roskies and H. Thacker, Phys. Rev. D60, 054505(1999).

3. A. Duncan, E. Eichten and H. Thacker, Phys. Rev. D 63, 111501 (2001) arXiv:heplat/0011076.

4. M. Alford, W. Dimm, G.P. Lepage, G. Hockney, and P.B. Mackenzie, Phys. Lett. B361, 87(1995).

5. H. Leutwyler and A. Smilga, Phys. Rev. D46, 5607 (1992).

6. See for example: www.phoenixcollective.org. 